\title{
LA POLÍTICA CHILENA DE ELECCIÓN DE COLEGIOS AL PIZARRÓN
}

Alejandra Mizala Salces 


\section{ALEJANDRA MIZALA SALCES}

Economista de la Universidad de Chile y $\mathrm{PhD}$ en Economía de la Universidad de California, Berkeley. Profesora Titular de la Facultad de Ciencias Físicas y Matemáticas. Actualmente es Directora del Departamento de Ingeniería Industrial y Directora Académica del Centro de Investigación Avanzada en Educación de la Universidad de Chile. 


\section{LA POLÍTICA CHILENA DE ELECCIÓN DE COLEGIOS AL PIZARRÓN}

\section{INTRODUCCIÓN}

Hasta comienzos de los años ochenta existían tres tipos de establecimientos educacionales en Chile: públicos, que respondían al 78\% de la matrícula escolar; privados, que recibían una subvención del Estado, con un 15\% de la matrícula; y privados pagados, con 7\% de la matrícula. Tanto los establecimientos públicos como los privados que recibían subvención estatal eran gratuitos y financiados por el Estado. Estos últimos recibían un subsidio equivalente a un monto que era la mitad del costo por alumno en el sector público; la mayoría de ellos eran católicos (Aedo 2000). Los colegios privados pagados cobraban a los padres y atendían a la elite del país.

En el año 81, durante la dictadura militar se implementaron importantes reformas en el sistema educacional del país. Estas reformas se focalizaron en la descentralización, privatización y generación de competencia del sistema educacional. En particular, apuntaron a permitir que empresas o personas particulares (llamadas “sostenedores") pudieran crear y administrar establecimientos educacionales, financiados con recursos públicos (denominados establecimientos particulares subvencionados). Al mismo tiempo se descentralizó la gestión de los establecimientos públicos desde una entidad central, Ministerio de Educación, a las municipalidades (establecimientos municipales). Uno de los elementos claves de esta reforma fue el financiamiento estatal a los establecimientos educacionales vía una subvención por alumno; subvención que es idéntica para establecimientos municipales y particulares subvencionados.

En efecto, la reforma de los ochenta buscó crear un mercado de servicios educacionales para las familias y para ello cambió el sistema de financiamiento desde un financiamiento directo al colegio por un financiamiento vía subvención, que corresponde a un esquema de subsidios educacionales donde los fondos siguen a los niños. Producto de esto se creó un sistema donde coexisten tres tipos de establecimientos educacionales: municipales, particulares subvencionados y particulares pagados. La apuesta era que la competencia entre los establecimientos educacionales por alumnos promovería la eficiencia en la educación chilena. 
Esta reforma permitió la creación de un gran número de colegios privados dispuestos a recibir la subvención estatal por alumno, lo que generó una importante migración de estudiantes desde los colegios públicos a este nuevo tipo de colegio. Ya en 1990 los colegios particulares subvencionados respondían por un 32\% de la matrícula escolar a expensas del sector público, que alcanzaba un 58\% de la matrícula. En el año 2012 los colegios particulares subvencionados alcanzaron un 53\% de la matrícula, mientras que los públicos/municipales un 37\%. ${ }^{1}$ Esta situación deja a Chile como uno de los sistemas escolares con menor participación de la educación pública, lo que contrasta con la tendencia que muestran los países desarrollados; efectivamente, un $89 \%$ de la matrícula primaria y un $81 \%$ de la matrícula secundaria (upper secondary) asiste a establecimientos públicos en los países de la OECD. ${ }^{2}$

Los estudiantes que han migrado desde el sector público al particular subvencionado pertenecen, en promedio, a familias de mayor nivel socioeconómico que aquellos que han permanecido en el sector público (Torche 2005, Hsie y Urquiola 2006).

Los establecimientos particulares pagados prácticamente no se vieron afectados por esta transformación. Ellos han continuado educando a un relativamente pequeño grupo de familias de mayor nivel socioeconómico que constituyen la elite del país.

Es importante hacer notar que el único requisito que se estableció para recibir la subvención fue la asistencia de alumnos a clases, sin instituir requisitos de calidad y otros. En particular, se podrían haber establecido requisitos de no discriminación, información de los resultados educativos a la comunidad, requisitos de calidad mínima, es decir, que los alumnos no sólo asistan sino que aprendan. Algunas de estas exigencias están presentes en otras experiencias internacionales en que el Estado financia por igual a establecimientos públicos y privados, ver Patrinos, Barrera-Osorio y Guaqueta (2009), Karsten (1999) y Ahlin (2003) para información de los casos de Holanda y Suecia.

En suma, se trata de un enfoque que ve el mecanismo de mercado y la competencia como una forma efectiva de incrementar la calidad de las escuelas. Precisamente porque se basa en la premisa de que la educación no es diferente a cualquier otro bien o servicio y que, por tanto, su calidad depende principalmente de que exista competencia, la idea fuerza detrás de la política era que la subvención, la entrada de proveedores privados, y la libre entrada y salida de colegios debían

1 Para detalles de la evolución de la matrícula que asiste a los distintos tipos de establecimientos educacionales en el país ver Gráfico 1 en el Anexo.

2 Para detalles por países ver Gráfico 2 en el Anexo. 
ser los principales mecanismos que asegurarían el funcionamiento eficiente del sistema.La políticas educacionales pueden ser evaluadas en función de al menos tres elementos: gobernanza, acceso y equidad, y propósito público de la educación. En lo que sigue de este trabajo se examinará, a la luz de estos elementos, la experiencia de Chile con la política de elección de colegios implementada a partir del año 1981.

\section{GOBERNANZA/REGULACIÓN}

Por gobernanza se entiende el que un sistema educacional asegure eficacia y calidad. En Chile, como ya se mencionó, el sistema de libre elección escolar -en su origenrespondió a un enfoque que priorizó la creación de un mercado educativo. La idea fuerza detrás de esta política, implementada durante el gobierno de Pinochet, era que las fuerzas del mercado por sí solas lograrían mejorar la calidad de la educación, la competencia entre escuelas (de gestión privada y pública) por estudiantes y la libre entrada y salida de colegios producirían un sistema escolar eficiente. Sin reconocer la complejidad del sistema educativo.

Sin embargo, este sistema no asegura calidad ni equidad de la educación, en gran medida porque el esquema de libre mercado no funciona adecuadamente en educación. Hay fallas de mercado propias de un sistema educativo, tales como asimetrías de información y problemas de agencia, que impiden que el sistema por sí solo genere educación de calidad. La calidad de la enseñanza no es observable directamente, lo que se agrava por su carácter multiproducto; esto es, la educación no sólo busca que los niños aprendan lenguaje y matemáticas, sino que se conviertan en ciudadanos formados integralmente, conscientes y responsables.

En efecto, la dificultad de observar la calidad de la educación impartida por una escuela genera asimetrías de información entre los que ofrecen el servicio educativo (colegios) y quienes lo demandan (padres y apoderados), lo que, de no ser resuelto adecuadamente, incentiva la mala calidad, en la medida que producir una educación de alta calidad tiene un mayor costo.

Al mismo tiempo, la falla de información genera problemas de agencia. Se habla de problemas de agencia cuando un individuo (principal) encarga un trabajo a otro (agente), en circunstancias que, por una parte, el principal no tiene toda la información necesaria respecto del esfuerzo y la calidad del trabajo del agente (asimetrías de información) y, por otra parte, pueden existir diferencias entre los objetivos que persigue cada uno de los individuos. Más aún, en educación existe un modelo de agente-principal donde hay más de un principal (Ministerio de Educación, sostenedor, director) y muchos agentes; además, algunos actores son agentes o principales dependiendo del punto de vista desde el cual se haga el análisis. Este es 
el caso, por ejemplo, de los directores de establecimientos, los que son principales en su relación con los docentes del establecimiento que dirigen y agentes en sus relaciones con los sostenedores y el Ministerio de Educación.

Además, el sistema genera discriminación y segmentación social de los alumnos, alterando el rol de la educación en la igualación de capacidades. Dado que el resultado observado del proceso educativo depende de las características de los alumnos, se genera una dinámica asociada a la llamada "competencia-S", es decir, existen incentivos para mejorar el resultado promedio de un colegio excluyendo a los alumnos de menor rendimiento potencial o real (González, 2000).

Consistente con la visión de mercado, en los años ochenta la política educacional no se focalizó en la escuela y sus necesidades. Más aún, durante el gobierno de Pinochet disminuyó significativamente el gasto en educación, de hecho, a comienzos de los años noventa el sistema estaba muy desfinanciado, la subvención por alumno había caído un 29\% en términos reales entre 1981 y 1990, y los salarios de los profesores habían caído 32\% en términos reales en el mismo periodo.

Solo al llegar la democracia el año 1990 comenzaron a implementarse programas de mejoramiento escolar. Con este fin se desarrollaron programas de mejoramiento y renovación pedagógica: Programas de apoyo focalizado a las escuelas más pobres (P-900; 1990), MECE básica (1992) y media (1995), básica rural, Proyecto Montegrande (1997), Enlaces (1997), Fortalecimiento de la profesión docente (formación inicial, pasantías en el extranjero; 1996-97), Liceo para todos (retención en enseñanza media; 2000).

Asimismo, se desarrollaron políticas orientadas a alterar las condiciones políticas, financieras y laborales, entre ellas: establecimiento del Estatuto Docente (1991) y sus posteriores cambios con el objeto de flexibilizarlo (1994 y 2000), y aumentos constantes del presupuesto del Ministerio de Educación.

Luego, se implementaron políticas orientadas a entregar incentivos monetarios ligados a la evaluación del desempeño docente: incentivos colectivos al desempeño docente (SNED, 1996), incentivos individuales (asignación de excelencia pedagógica, red maestro de maestros, 2001) y evaluación del desempeño individual (sólo para la educación municipal, 2001).

Se articuló una reforma que añadió a estos programas una reforma curricular, la extensión de la jornada escolar (1997) y los 12 años de enseñanza obligatoria (2001).

Como puede observarse, en gran medida las políticas de los gobiernos de la Concertación enfatizaron el aumento y la mejora de la calidad de los insumos educativos, pero fueron tímidas respecto del establecimiento de normas, mayor regulación e incentivos para que el sistema educacional funcione en forma adecuada en un esquema de financiamiento vía subvenciones. 
Recién luego del movimiento (pingüino) liderado por los estudiantes secundarios el año 2006 se comenzó a discutir en el país la necesidad de una mayor regulación. Sólo a partir de entonces se empezó a implementar un sistema de aseguramiento de la calidad de la educación escolar, el que ha venido de la mano de la Ley General de Educación del año 2009 y más recientemente en el año 2011 de la creación de la Agencia de la Calidad y la Superintendencia de Educación, que junto al Consejo Nacional de Educación establecen una nueva arquitectura del sistema escolar.

Esta falta de regulación y otras fallas de diseño llevaron a un sistema educativo con resultados deficientes en las comparaciones internacionales. Los resultados de Chile en las pruebas internacionales como PISA y TIMSS muestran que Chile está entre los países de menor desempeño en estas pruebas, lo que no puede asociarse con el carácter descentralizado de su sistema educacional, ya que hay países como Nueva Zelandia, Suecia u Holanda que teniendo un sistema descentralizado obtienen resultados que los ubican en los mejores puestos entre los países que rinden estas pruebas.

Asimismo, los resultados en las pruebas nacionales SIMCE muestran un preocupante estancamiento de los resultados y no se observan mejoras relevantes en los resultados educacionales, al menos en los aspectos que miden las pruebas estandarizadas de logro educacional. ${ }^{3}$ También se observa una significativa brecha por tipo de establecimiento educacional, que responde a las diferencias socioeconómicas de la población que atienden.

En efecto, en términos del desempeño de los establecimientos públicos/ municipales versus los particulares subvencionados, los estudios más recientes y con mejores datos y metodologías encuentran que los establecimientos particulares subvencionados no tienen mejores resultados que los públicos/municipales, una vez que se corrige por las características de sus estudiantes. Usando una serie de técnicas econométricas que permiten comparar estudiantes con similares características, Lara, Mizala y Repetto, (2011) encuentran que la educación privada subvencionada tiene sólo un pequeño impacto - de dos a tres puntos, esto es, entre 4\% y $6 \%$ de una desviación estándar-, no siempre estadísticamente significativo, en los resultados académicos medidos por la prueba SIMCE.

\section{EQUIDAD Y ACCESO}

Cuando se evalúa un sistema educacional desde el punto de vista de acceso y equidad hay dos elementos a considerar: el financiamiento y las políticas de admisión. El

Los Gráficos 3 y 4 en el Anexo presentan estos resultados. 
objetivo es que cada niño y joven tenga acceso a los recursos educativos que necesita para desarrollarse.

\section{Política de financiamiento}

En Chile la política de financiamiento en su diseño original estableció una subvención pareja por alumno, sin reconocer que alcanzar un determinado nivel de aprendizaje requiere que los recursos varíen en forma inversa al nivel socioeconómico de las familias. Esto genera incentivos para seleccionar alumnos de mayor nivel socioeconómico, discriminar a los más pobres, porque tienen menor resultado potencial, y expulsar a aquellos con menor rendimiento. Esta decisión no consideró la evidencia internacional, que sugiere una relación inversa entre el aprendizaje y el nivel socioeconómico o educacional de los adultos del hogar. Niños y jóvenes con mayor desventaja económica tienen, en general, más dificultades que superar en el proceso de aprendizaje y, por lo tanto, su educación es más demandante en términos de recursos (Ducombe y Yinger, 2000; Reschovsky y Imazeki, 2001).

Por tanto, los establecimientos educacionales han tenido incentivos para conseguir buenos alumnos con padres de mayor nivel socioeconómico, en vez de únicamente esforzarse por entregar buena educación. Esto ha dificultado que el sistema escolar promueva una educación de alta calidad para todos los niños y jóvenes (Akiba et al 2007). Al mismo tiempo se producen significativas desigualdades socioeconómicas en los resultados educativos y segregación entre sectores educacionales y entre los distintos establecimientos al interior de ellos (Torche 2005; Hsieh y Urquiola 2006; Mizala y Torche 2012, Elacqua 2012).

Para corregir este error de diseño el año 2008 se estableció la Subvención Escolar Preferencial (SEP), que otorga una subvención mayor a aquellos niños de menor nivel socioeconómico y que cubre al $40 \%$ más pobre de la población estudiantil. Además, se entregan recursos adicionales de acuerdo a la proporción de alumnos prioritarios que tenga el establecimiento escolar. Los establecimientos públicos y privados que reciben subvención del Estado pueden voluntariamente participar en esta política, para lo cual deben adquirir una serie de compromisos de orden académico y administrativo. Es importante notar que esta es la única subvención que está condicionada al cumplimiento de requisitos, metas institucionales y resultados. ${ }^{4}$ Los estudios existentes indican que hay un efecto positivo de esta

4 La participación en la SEP es voluntaria y el sostenedor debe firmar un Convenio de Igualdad de Oportunidades a través del cual el establecimiento se compromete a implementar un Plan de Mejoramiento Educativo, mejorar sus resultados educacionales (medidos a través de la prueba 
política sobre los resultados académicos de los colegios adscritos al sistema. La SEP obtiene resultados alineados con sus objetivos; esto es, logra mejorar los resultados educativos, medidos por las pruebas estandarizadas de logro, fundamentalmente de los establecimientos educacionales que atienden a la población de menor nivel socioeconómico, con lo cual contribuye a reducir la brecha socioeconómica en los resultados educacionales (Correa et al 2013; Mizala y Torche 2013; Valenzuela et al 2013; Nielsen 2014).

Pero el sistema chileno no sólo ha tenido una subvención pareja por estudiante, sino que además los colegios particulares subvencionados pueden cobrar a los padres un monto por sobre la subvención que el establecimiento educacional recibe del Estado. Esto diferencia el caso de Chile de todas las otras experiencias internacionales con sistemas de libre elección de colegios. Esta política, llamada de financiamiento compartido, restringe la libertad de elección de las familias, condicionando la elección del colegio en el cual desean educar a sus hijos a su capacidad de pago (Flores y Carrasco, 2013), sin generar mejores resultados educativos como muestran estudios recientes.

Estimar el efecto del financiamiento compartido sobre los resultados académicos no es una tarea sencilla. Esto, porque la simple comparación de puntajes promedio en la prueba SIMCE entre los estudiantes que asisten a establecimientos con y sin financiamiento compartido no produce resultados válidos, debido a que los niños que asisten a colegios con financiamiento compartido tienen características diferentes de los que van a colegios gratuitos. Asimismo, los colegios que optan por cobrar financiamiento compartido son diferentes en diversas dimensiones de los que deciden no hacerlo. Por lo tanto, es necesario utilizar métodos estadísticos que eliminen, o al menos minimicen, el sesgo de selección asociado a no estar comparando niños y colegios con exactamente las mismas características. En un estudio que busca evaluar el efecto de esta política sobre los resultados educativos Mizala y Saavedra (2014) se concentran en los establecimientos particulares subvencionados, ya que muy pocos colegios municipales de enseñanza media cobran a los padres y los que lo hacen solicitan un pago muy reducido.

Los datos muestran que los padres cuyos hijos asisten a establecimientos particulares subvencionados con financiamiento compartido tienen, en promedio, mayores ingresos, niveles más altos de educación, más recursos para estudiar en casa (libros, computador, etc.) y expectativas más altas sobre la educación que alcanzarán

SIMCE), a no seleccionar y retener a los alumnos, y no cobrar financiamiento compartido a los alumnos prioritarios. 
sus hijos, en comparación con los padres cuyos hijos asisten a establecimientos particulares subvencionados gratuitos.

Otro elemento importante de este estudio es que toma en cuenta la presencia de efectos heterogéneos, es decir, se evalúa cómo varía el efecto del financiamiento compartido sobre el rendimiento escolar, según los años que el colegio lleva en el sistema y el monto que cobra a los padres.

Los resultados obtenidos son robustos, ya que se utilizaron diferentes técnicas econométricas y con los distintos estimadores se obtuvieron resultados similares, y permiten establecer que el financiamiento compartido tiene un efecto positivo sobre el rendimiento escolar sólo cuando el colegio lleva más de nueve años cobrando a los padres y cuando el monto es superior a $\$ 8.000$ mensuales. Sin embargo, estos beneficios de largo plazo sólo se ven reflejados en los puntajes de matemáticas, pues en lenguaje no se aprecian diferencias entre los colegios que cobran y los gratuitos, independiente de los años que el establecimiento esté en el sistema y del monto que pida. ${ }^{5}$

Por su parte, los colegios particulares subvencionados que llevan menos de 10 años con financiamiento compartido no tienen mejores resultados académicos que los gratuitos (ni en matemáticas ni en lenguaje). Más aún, es importante señalar que gran parte de las diferencias en rendimiento escolar entre los alumnos que asisten a establecimientos particulares subvencionados con financiamiento compartido y los que asisten a establecimientos gratuitos, son atribuibles a diferencias en las características de los alumnos y de sus compañeros de curso. Cuando se compara a estudiantes y cursos con características similares el efecto disminuye considerablemente en matemáticas y desaparece completamente en lenguaje. Esto significa que el efecto sobre los resultados escolares del financiamiento compartido opera fundamentalmente vía la homogeneización de la sala de clases.

En suma, lo que el sistema de financiamiento compartido hace es escoger a los niños a través de la capacidad de pago de sus padres y ponerlos juntos en la misma sala de clases. Así, los resultados obtenidos en el SIMCE no se deben a que los colegios agreguen más valor, sino a que los niños tienen ventajas de origen que les permiten rendir mejor. Estamos, por tanto, en un mundo en que los padres escogen colegios de acuerdo a su capacidad de pago y los colegios, en vez de esforzarse por entregar buena educación, tienen incentivos para conseguir alumnos con padres de mayor nivel socioeconómico.

5 Para mayores detalles de las estimaciones ver Cuadros 1 y 2 en el Anexo. Resultados similares han sido obtenidos por Paredes et al (2013). 
Asimismo, seha encontrado que el financiamientocompartidoestá correlacionado con la segregación socioeconómica. Valenzuela, Bellei y De Los Ríos (2014) hallan que mientras mayor es el número de escuelas particulares subvencionadas que cobran financiamiento compartido, mayor es el nivel de segregación socioeconómica en las escuelas de la comuna y Elacqua (2012) muestra que las escuelas con financiamiento compartido matriculan una menor proporción de estudiantes vulnerables que aquellas gratuitas y municipales. El financiamiento compartido es sospechoso de intensificar a nivel del sistema escolar la segmentación residencial que existe en el país. Un estudio de Elacqua y Santos (2013) compara el nivel de segregación escolar actual -usando para ello distintos índices de segregación desarrollados en la literatura empírica- con aquel que existiría bajo el escenario contrafactual en que los estudiantes asisten al establecimiento educacional (municipal o particular subvencionado) más cercano a su lugar de residencia; ellos encuentran que el nivel de segregación en el escenario en que los niños van al establecimiento más cercano a su hogar es menor que el nivel de segregación actual, es decir, los establecimientos educacionales están más segregados socioeconómicamente que los barrios.

\section{Políticas de admisión}

Cuando hay libre elección de colegios por parte de los padres es fundamental impedir la selección de estudiantes por parte de los establecimientos educacionales, si se permite la selección y prima la competencia las escuelas tendrán incentivos para elegir a los estudiantes de mayor nivel socioeconómico y sin problemas de aprendizaje. Si esto ocurre las escuelas se diferencian más por su capacidad para escoger sus estudiantes que por el valor que agregan. También se plantea que si hay libre elección de colegios, se deberá garantizar que todos los miembros de la sociedad puedan hacer una auténtica elección, para lo que se requiere información veraz y precisa sobre el desempeño académico de las escuelas.

Chile no cumple con ninguna de estas consideraciones. Hasta el año 2009 los colegios particulares subvencionados eran libres de seleccionar a sus alumnos. Hoy día, si bien la Ley General de Educación de 2009 impide la selección hasta $6^{\circ}$ básico, existe evidencia que muestra que los colegios particulares subvencionados aún seleccionan a sus alumnos a través de exámenes de admisión y entrevistas a los padres (Godoy, Salazar y Treviño, 2014).

Por otra parte, hay un enorme desafío respecto de la información, ya que si bien a los padres sí les importa la calidad de los colegios, medida ésta por los resultados de pruebas estandarizadas, los resultados de estas pruebas están muy correlacionados con el nivel socioeconómico de los estudiantes y, por tanto, no miden adecuadamente 
la efectividad de los colegios. Sin embargo, el tema es complejo, porque los padres no necesariamente responden a información sobre efectividad de los colegios, ya que a muchos les importa la composición socioeconómica del colegio de sus hijos, esto quiere decir que hay autoselección. Por ello entregar información sobre efectividad escolar no necesariamente resuelve el problema de la elección de colegios efectivos en Chile (Mizala y Urquiola, 2013).

\section{III.PROPÓSITO PÚBLICO DE LA EDUCACIÓN}

El propósito público de la educación se refiere a que las escuelas deben cultivar en todos los estudiantes destrezas y conocimientos que les permitan pensar en forma independiente, vivir juntos productivamente y compartir los valores de una democracia pluralista. Lo cierto es que no hay nada en el sistema de libre elección de colegios que impida cumplir con el propósito público de la educación, sin embargo, debe haber reglas y mecanismos que comprometan a todos los establecimientos educacionales con estos objetivos.

Para esto no basta la rendición de cuentas basada en pruebas estandarizadas, se requiere padres más involucrados y que todos los establecimientos educacionales rindan cuentas señalando en sus propuestas cómo cumplirán los objetivos públicos de inclusión y ciudadanía. Con este fin es necesaria la supervisión por parte de agencias que tengan capacidad de monitoreo efectivo y que estén comprometidas con los objetivos públicos de no selección e inclusión.

¿Estamos en Chile cumpliendo con el propósito público de la educación? Es difícil que así sea.

La capacidad de seleccionar de los colegios particulares subvencionados, la subvención pareja hasta el año 2008 y el financiamiento compartido han generado un sistema educacional con una forma particular de estratificación. Contrariamente a la visión simplificada, en la cual los colegios particulares subvencionados atraerían a los estudiantes de más alto nivel socioeconómico de los colegios públicos/ municipales, dejando a los más pobres en estos colegios, lo que los datos muestran es que el sistema particular subvencionado atiende a familias de un amplio espectro socioeconómico, pero al interior de cada uno de los establecimientos el nivel socioeconómico de los estudiantes es muy homogéneo.

Esto implica que el sector particular subvencionado se ve inclusivo, porque hay todo tipo de colegios, es decir, desde colegios para clase media acomodada hasta colegios para pobres. Pero la unidad de análisis relevante no es el sector a nivel agregado, sino el colegio. En Chile los colegios particulares subvencionados estratifican por nivel socioeconómico y consecuentemente por resultados 
académicos; hay colegios particulares subvencionados para niños acomodados y con buenos resultados escolares, y colegios para pobres con peores resultados académicos.

Lo anterior se grafica claramente en el hecho que la variabilidad del indicador de estatus socioeconómico (medido por el ingreso del hogar y la educación de los padres) entre colegios es de un $24 \%$ en el sector público/municipal, en tanto que en el sector particular subvencionado llega a 47\% (Mizala y Torche, 2012). Otra forma de graficar el hecho de que los colegios particulares subvencionados son más estratificados que los colegios municipales es la correlación que existe entre el colegio y el ingreso del hogar de sus alumnos; en el sector particular subvencionado esta correlación es el doble que la que existe en el sector municipal. Un resultado que significa que basta conocer el colegio donde un niño estudia en el sector particular subvencionado para tener información muy acertada del ingreso de su familia.

Esta estratificación socioeconómica tiene un correlato directo con la estratificación de los resultados escolares, medido por pruebas estandarizadas tipo SIMCE. De hecho, la variabilidad de los puntajes de esta última prueba entre colegios particulares subvencionados es mucho mayor $(27 \%)$ que la variabilidad que se obtiene entre colegios municipales (14\%), un resultado que se repite en pruebas de diferentes materias (lenguaje y matemáticas), así como en distintos grados. Esto significa que existe una gran estratificación de puntajes en las pruebas SIMCE en el sector particular subvencionado (Mizala y Torche, 2012). Una forma de interpretar este resultado es la siguiente: es más probable que el peor estudiante de un buen colegio tenga mejores resultados que el mejor estudiante de un colegio malo en el sector particular subvencionado que en el sector municipal. Esto significa que en el sector particular subvencionado el colegio también es una unidad de estratificación del desempeño escolar.

La pronunciada estratificación económica del sector particular subvencionado también se manifiesta en la elevada asociación que existe entre el nivel socioeconómico del colegio (que es el promedio del nivel socioeconómico de todos los alumnos) y los resultados en el SIMCE. Esta asociación es más del doble en tamaño en los colegios particulares subvencionados que en los colegios municipales, aun después de aislar el impacto del nivel socioeconómico individual. En otras palabras, el resultado educativo de un estudiante que asiste a un colegio particular subvencionado depende en gran medida de la composición socioeconómica del alumnado del colegio al que va, lo que significa que nuestras aulas no tienen la capacidad de interrumpir la reproducción cultural de la pobreza (Mizala y Torche, 2012).

Estamos, por lo tanto, en un mundo en que los padres escogen colegios de acuerdo a su capacidad de pago y a los resultados promedio del establecimiento y los colegios, en vez de esforzarse por entregar buena educación, tienen incentivos 
para conseguir "buenos" alumnos con padres de mayor nivel socioeconómico, lo que impide que el sistema escolar promueva una educación de alta calidad para todos los niños y jóvenes.

Pero hay otras razones por las que el fuerte grado de homogeneidad interna de los colegios en Chile es preocupante. Esta realidad implica que niños de diferentes contextos sociales y culturales no tienen la posibilidad de interactuar, lo que es en sí mismo malo para la democracia, más allá de lo que ocurra con los resultados escolares. Idealmente la escuela debiera ser una instancia de aprendizaje con los demás y acerca de los demás, de forma de educar en el respeto de los derechos del otro, desarrollando las virtudes cívicas sobre las que descansa la democracia.

\section{COMENTARIOS FINALES}

Nuestro desafío como país es crear un sistema con muchas escuelas dignas de elegir, que estén abiertas para todos, escuelas que preparen a las futuras generaciones para participar en la vida política -a través de una adecuada educación cívica-, social y económica del país.

Para cumplir con este desafío una condición necesaria es fortalecer la educación pública para que los padres vuelvan a elegirla, solo así lograremos disminuir significativamente la segmentación social de nuestro sistema educacional. Ello requiere resolver los problemas organizativos-institucionales que ella enfrenta y equilibrar las reglas del juego a las que están sometidos los establecimientos públicos y particulares subvencionados.

La dificultad principal que enfrenta la educación pública es que su esquema organizativo-institucional no tiene dirección clara. La forma como se implementó la descentralización del sistema educacional está en la raíz de los problemas que afectan hoy en día al sistema público educativo y que dificultan la adecuada gestión del mismo. Se da una situación anómala en que los municipios están a cargo de la gestión administrativa y el Ministerio de Educación mantiene la responsabilidad por los aspectos técnico-pedagógicos. Como consecuencia de esto se diluye la responsabilidad de los municipios por los resultados educativos de sus colegios y se observa una mirada ministerial distante a los problemas de gestión de los municipios.

Adicionalmente, la relación entre la educación pública y la educación privada subvencionada no es ecuánime, lo que también ha afectado negativamente a la educación pública. Hay diferencias en la capacidad de seleccionar a sus estudiantes, en el acceso a fuentes de financiamiento, particularmente el financiamiento compartido, en los costos asociados a atender a una población con mayores grados de vulnerabilidad y en las regulaciones a las que ambas están sujetas. 
El gobierno de la Presidenta Bachelet ya envió al Congreso un proyecto de ley que propone terminar con la selección, el financiamiento compartido y el lucro en la educación que se financia con fondos públicos. Con esto Chile se pone en línea con las prácticas mejor establecidas en el mundo desarrollado, asegurando que todos los establecimientos educacionales, independiente de su carácter público o privado, cumplan el propósito público de la educación. La tarea pendiente es convertir a la educación pública en el eje del sistema escolar, que garantice el derecho constitucional de todos -social y geográficamente- a la educación obligatoria y gratuita; que asegure la existencia de proyectos educativos integradores; y que sea capaz de fijar un elevado estándar de calidad, promoviendo la búsqueda de la excelencia en el conjunto del sistema educativo.

\section{REFERENCIAS}

Aedo, R. (2000). La educación privada en Chile: Un estudio histórico-analítico desde el periodo colonial hasta 1990. Santiago: RIL Editores.

Ahlin, A. (2003). "Does school competition matter? Effects of a large-scale school choice reform on student performance". Mimeo Department of Economics Uppsala University.

Akiba, M. G. Le Tendre y J. Scribner ( 2007). “Teacher Quality, Opportunity Gap, and National Achievement in 46 Countries". Educational Researcher 36(7): 369-387.

Correa, J., Inostroza, D., Parro, F., Reyes, L. y G. Ugarte (2012). "El impacto del voucher diferenciado en los resultados académicos: el caso chileno". Mimeo Ministerio de Hacienda.

Ducombe, W. y J. Yinger (2000). "Financing higher student performance standards: the case of New York State". Economics of Education Review 19, 363-386.

Elacqua, G. (2012). "The impact of school choice and public policy on segregation: Evidence from Chile". International Journal of Educational Development 32: 444-453.

Elacqua, G. y H. Santos (2013). "Los efectos de la elección escolar en la segregación socioeconómica en Chile: Un análisis georreferenciado". Documento de Referencia 1. Espacio Público.

Flores, C. y A. Carrasco (2013). "(Des)igualdad de oportunidades para elegir escuela: preferencias, libertad de elección, y segregación escolar”. Documento de Referencia 2. Espacio Público. 
Godoy, F., Salazar. F. y E. Treviño (2014).’Prácticas de selección en el sistema escolar chileno: requisitos de postulación y vacíos legales". Informes para la Política Educativa $N^{\circ} 1$, Centro de Políticas Comparadas de Educación, Universidad Diego Portales.

González, P. (2000). "Fallas del mercado y políticas públicas: aplicación a las políticas sociales". Serie Docente 22, CIEPLAN.

Hsieh, C. y M. Urquiola (2006). The effect of generalized school choice on achievement and stratification: evidence from Chile's school voucher program. Journal of Public Economics 90: 1477-1503.

Karsten, S. (1999). "Neoliberal education reform in the Netherlands", Comparative Education 35, N³.

Lara, B., Mizala, A. y A. Repetto (2011). "The Effectiveness of Private Voucher Education: Evidence from Structural School Switches”. Educational Evaluation and Policy Analysis 33(2): 119-137.

Mizala, A. y F. Torche (2012). "Bringing the Schools Back In: The Stratification of Achievement in the Chilean Voucher System". International Journal of Educational Development 32: 132-144.

Mizala, A. y M. Urquiola (2013). "School markets: The impact of information approximating schools' effectiveness". Journal of Development Economics 103: 313-335.

Mizala, A. y F. Torche (2013). “¿Logra la subvención escolar preferencial igualar los resultados educativos? Documento de Referencia 4. Espacio Público.

Mizala, A. y T. Saavedra (2014). "Efecto del financiamiento compartido sobre el rendimiento escolar". Mimeo Departamento de Ingeniería Industrial, Universidad de Chile.

Nielsen, C. (2014). "Targeted vouchers, competition among schools, and the academic achievement of poor students". Tesis de Doctorado, Universidad de Yale.

Paredes, R., Opazo, M., Volante, P. y J.R. Zubizarreta (2013). "Financiamiento compartido en la educación subvencionada chilena". Centro de Políticas Públicas UC, Propuestas para Chile, Pontificia Universidad Católica de Chile.

Patrinos, H. Barrera-Osorio, F. y J. Guaqueta (2009). The Role and Impact of Public-Private Partnerships in Education. Washington, DC: World Bank.

Reschovsky, A. y J. Imazeki. 2001. Achieving educational adequacy through school finance reform. Journal of Education Finance 26: 373-396.

Torche, F. 2005. "Privatization Reform and Equality of Educational Opportunity in Chile". Sociology of Education 78 (4): 316-343. 
Valenzuela, J.P., Villarroel, G. y C. Villalobos (2013). “Ley de Subvención Escolar Preferencial (SEP): algunos resultados preliminares de su implementación". Pensamiento Educativo. Revista de Investigación Educacional Latinoamericana $50(2)$.

Valenzuela, J.P., Bellei, C. y D. De Los Ríos (2014). "Socioeconomic school segregation in a market-oriented educational system. The case of Chile" Journal of Education Policy 29(2): 217-241. 


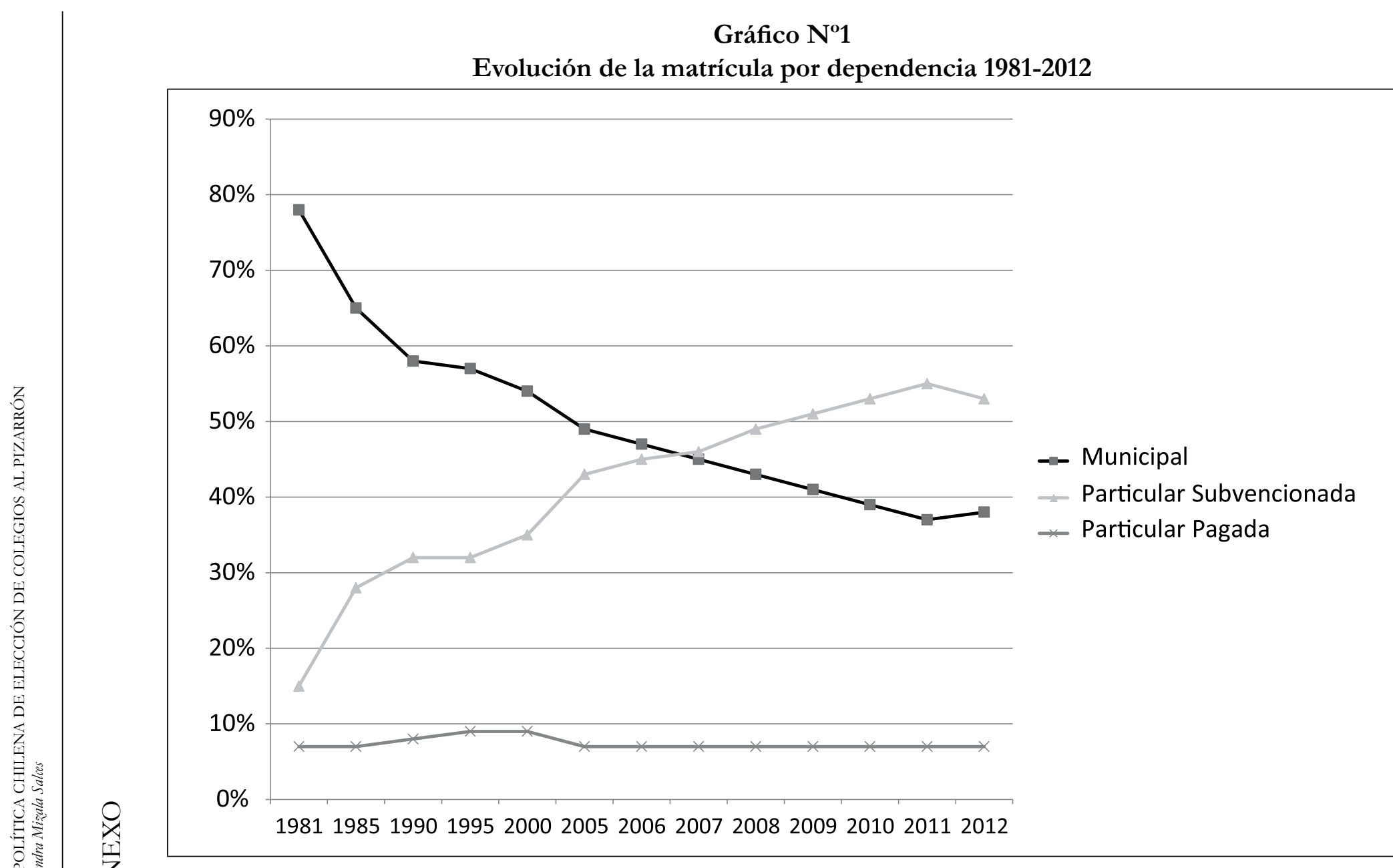




\section{Gráfico $\mathrm{N}^{\circ} 2$}

Matrícula que asiste a la educación pública en los países de la OECD. Educación primaria y secundaria año 2012. (Porcentajes)

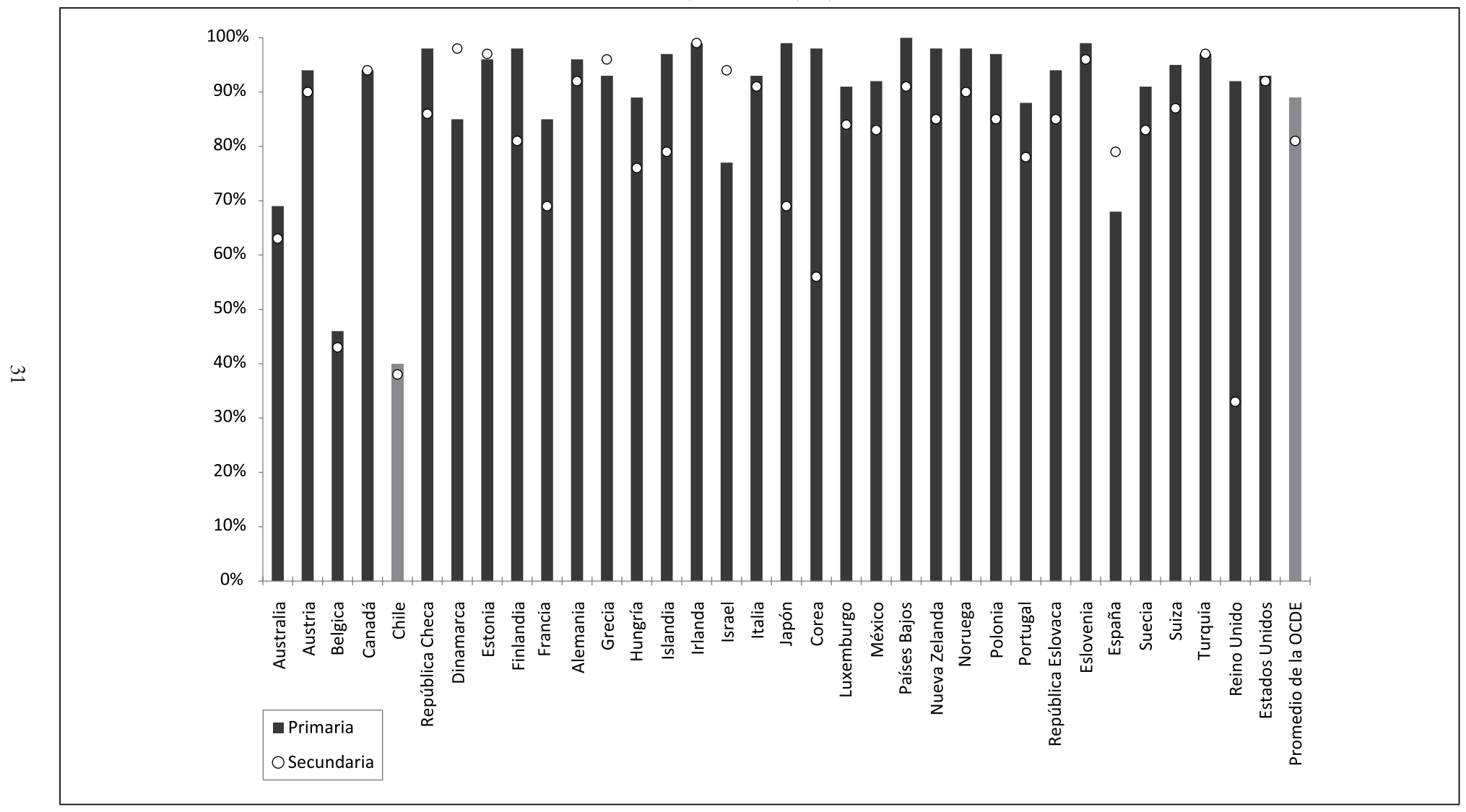

Fuente: Education at a Glance OECD (2014). 
Gráfico $\mathbf{N}^{\circ} 3$

Resultados Prueba SIMCE Lectura por dependencia $4^{\circ}$ Educación Básica

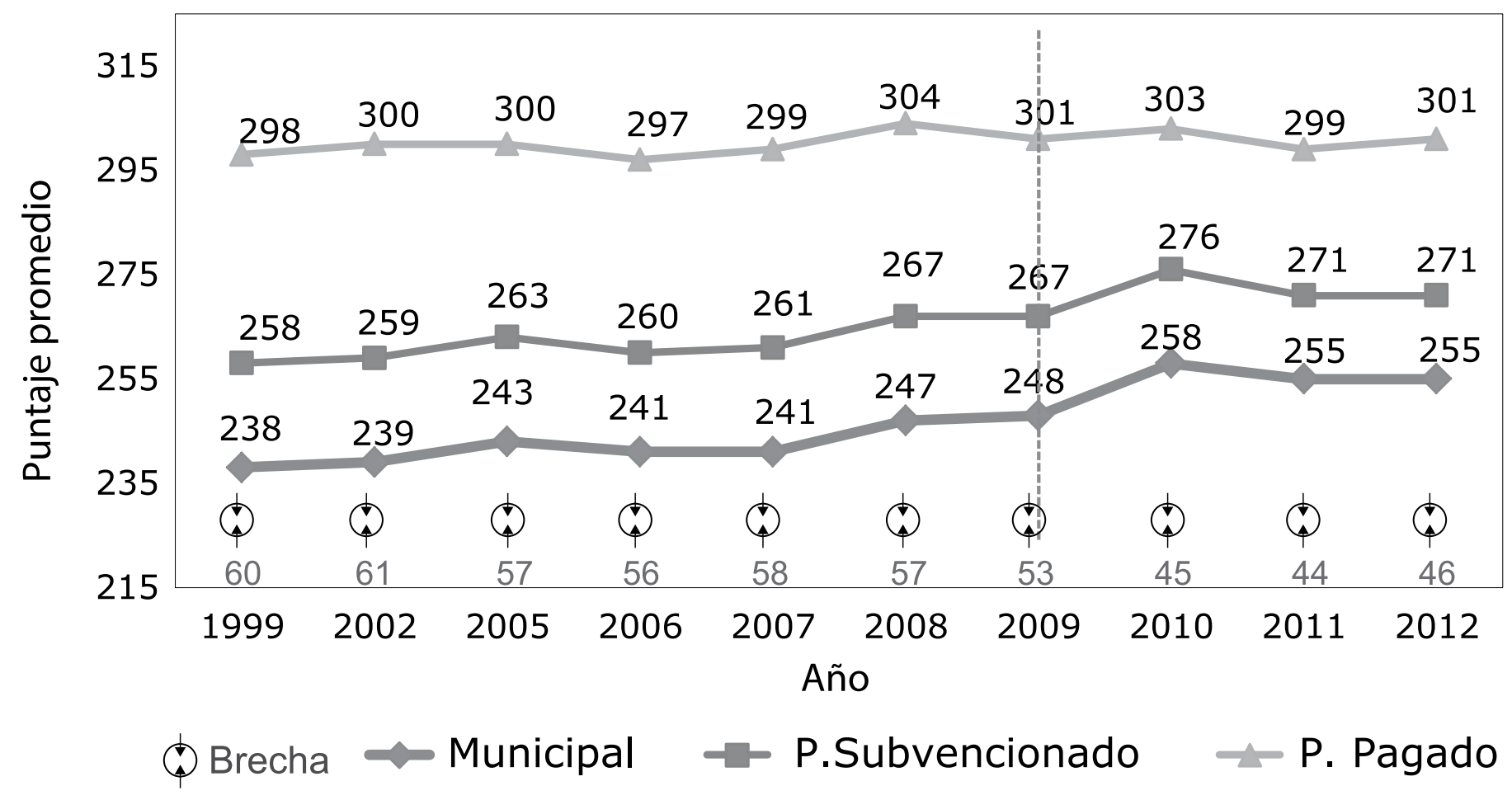




\section{Gráfico $\mathrm{N}^{\circ} 4$}

Resultados Prueba SIMCE Matemáticas por dependencia $4^{\circ}$ Educación Básica

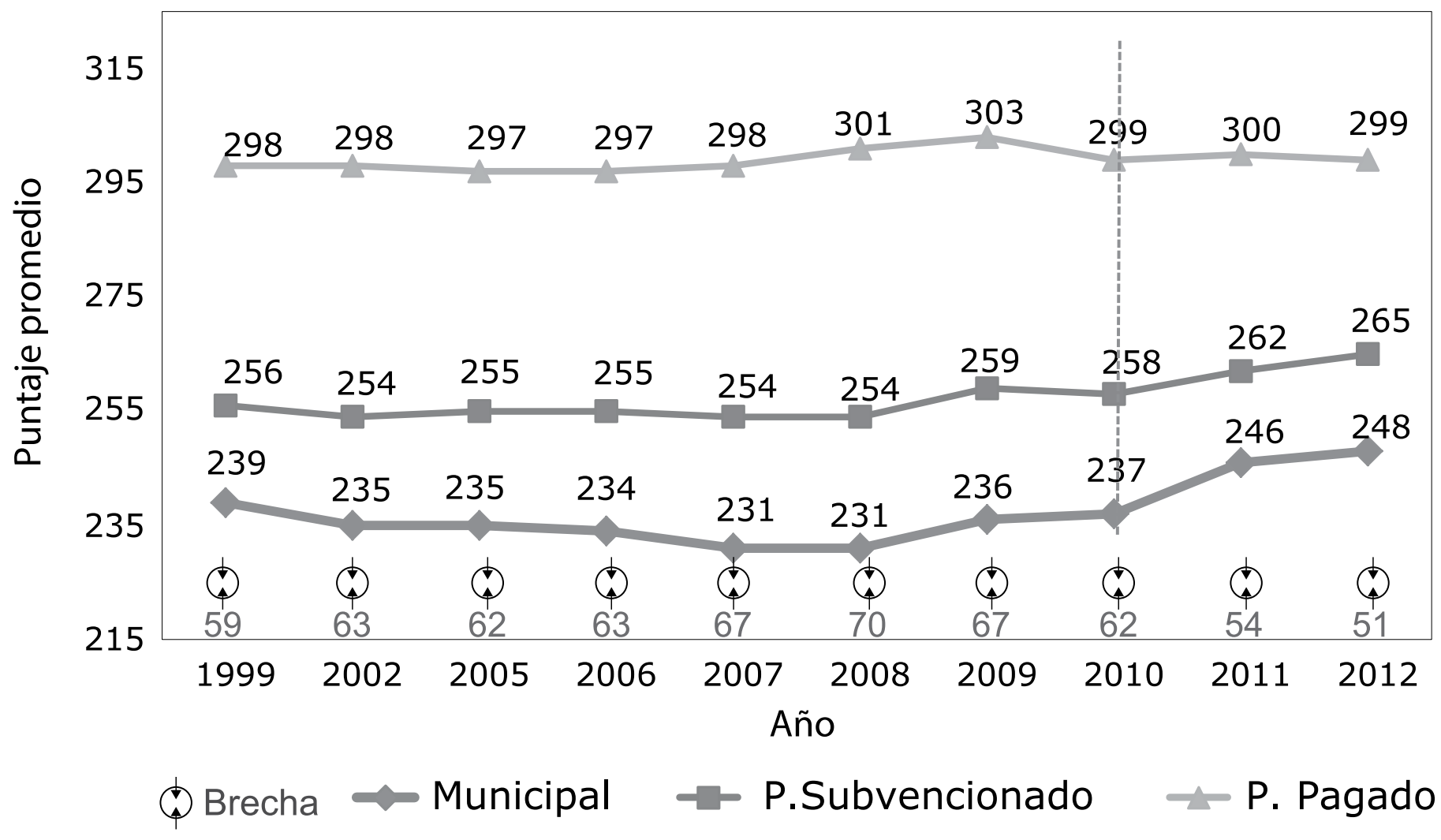




\section{Cuadro $\mathrm{N}^{\circ} 1$}

Efecto del financiamiento compartido en el SIMCE (según monto cobrado)

\begin{tabular}{|c|c|c|c|c|}
\hline \multirow[b]{2}{*}{ Grupo de Tratamiento } & \multicolumn{2}{|c|}{ Matemáticas } & \multicolumn{2}{|c|}{ Lenguaje } \\
\hline & PSW & DR & PSW & DR \\
\hline $\begin{array}{c}\text { Niños que en el año } 2009 \text { asisten a colegios que } \\
\text { tienen FICOM desde que se inició el sistema. } \\
\text { Quintiles de monto cobrado }\end{array}$ & $\begin{array}{c}6.9386^{* * *} \\
(2.0423)\end{array}$ & $\begin{array}{c}6.8415 * * * \\
(2.0109)\end{array}$ & $\begin{array}{c}2.7064 \\
(1.8562)\end{array}$ & $\begin{array}{c}2.6604 \\
(1.8294)\end{array}$ \\
\hline Primer quintil $[<\$ 4500]$ & $\begin{array}{l}-1.1228 \\
(2.8741)\end{array}$ & $\begin{array}{l}-1.3908 \\
(2.9006)\end{array}$ & $\begin{array}{l}-4.6014^{*} \\
(2.5482)\end{array}$ & $\begin{array}{l}-5.0261 * \\
(2.6016)\end{array}$ \\
\hline Segundo quintil [\$4600-\$7800] & $\begin{array}{c}3.7063 \\
(3.3254)\end{array}$ & $\begin{array}{c}3.4977 \\
(3.2262)\end{array}$ & $\begin{array}{c}0.5731 \\
(3.3899)\end{array}$ & $\begin{array}{c}0.3317 \\
(3.3306)\end{array}$ \\
\hline Tercer quintil [\$7810-\$12100] & $\begin{array}{l}5.8590^{*} \\
(2.6658)\end{array}$ & $\begin{array}{l}6.3021^{* *} \\
(2.7143)\end{array}$ & $\begin{array}{c}1.3584 \\
(2.5004)\end{array}$ & $\begin{array}{c}1.6776 \\
(2.5869)\end{array}$ \\
\hline Cuarto quintil [\$12200-\$20500] & $\begin{array}{l}11.6799^{*} \\
(6.8794)\end{array}$ & $\begin{array}{c}14.0252^{* *} \\
(6.6300)\end{array}$ & $\begin{array}{c}3.2672 \\
(8.7463)\end{array}$ & $\begin{array}{c}4.5357 \\
(8.3920)\end{array}$ \\
\hline Quinto quintil [\$20600-\$74600] & $\begin{array}{l}11.7629^{*} \\
(6.4404) \\
\end{array}$ & $\begin{array}{c}15.4516 * * \\
(6.7997) \\
\end{array}$ & $\begin{array}{c}5.2634 \\
(6.7234) \\
\end{array}$ & $\begin{array}{c}5.1032 \\
(8.1736) \\
\end{array}$ \\
\hline
\end{tabular}

\section{Cuadro $\mathrm{N}^{\circ} 2$}

\section{Efecto del financiamiento compartido en el SIMCE (según número de años)}

\begin{tabular}{|c|c|c|c|c|}
\hline \multirow{2}{*}{ Grupo de Tratamiento } & \multicolumn{2}{|c|}{ Lenguage } & \multicolumn{2}{|c|}{ Matemáticas } \\
\hline & PSW & $\mathrm{DR}$ & PSW & DR \\
\hline Niños que en el año 2009 asisten a colegios & $-0,400$ & $-0,691$ & $-1,872$ & -2.482 \\
\hline que tienen entre 1 y 4 años de FICOM. & $(3,291)$ & $(3,294)$ & $(4,484)$ & $(4,483)$ \\
\hline Niños que en el año 2009 asisten a colegios & $-1,823$ & $-1,255$ & 0,657 & 1,517 \\
\hline que tienen entre 5 y 8 años de FICOM. & $(2,687)$ & $(2,520)$ & $(2,964)$ & $(2,721)$ \\
\hline Niños que en el año 2009 asisten a colegios & 0,965 & 1,311 & $5,782 * *$ & $6,218 * * *$ \\
\hline que tienen entre 9 y 12 años de FICOM. & $(1,982)$ & $(1,899)$ & $(2,435)$ & $(2,331)$ \\
\hline Niños que en el año 2009 asisten a colegios & 2,180 & 2,106 & $5,760 * * *$ & $5,645 * * *$ \\
\hline que tienen entre 13 y 16 años de FICOM. & $(1,601)$ & $(1,555)$ & $(1,769)$ & $(1,694)$ \\
\hline \multicolumn{5}{|c|}{$\begin{array}{l}\text { Nota: El grupo control en todos los casos corresponde a alumnos asisten a establecimientos que nunca han tenido FC. } \\
\text { Errores estándar entre paréntesis. }\left({ }^{*}\right) \text { significativo al } 10 \%,(* *) \text { significativo al } 5 \%,(* *) \text { significativo al } 1 \% .\end{array}$} \\
\hline
\end{tabular}

Fuente: Mizala y Saavedra (2014). 